\title{
Perceptions et stratégies paysannes d'adaptation à la variabilité pluviométrique au Centre du Bénin
}

\author{
Joseph OLOUKOI $^{{ }^{*}}$, Ibouraima $\mathrm{YABI}^{2}$ et Christophe S. HOUSSOU ${ }^{2}$ \\ ${ }^{I}$ African Regional Institute for Geospatial Information Science and Technology (AFRIGIST), \\ Obafemi Awolowo University Campus, PMB 5545, Ile-Ife, Nigeria. \\ ${ }^{2}$ Laboratoire Pierre PAGNEY Climat, Eau, Ecosystèmes et Développement (LACEEDE), \\ Département de Géographie, Université d'Abomey-Calavi, BP 922 Abomey-Calavi, Bénin. \\ "Auteur correspondant; E-mail: chabijos@yahoo.fr, oloukoi@rectas.org.ng, chabijos@gmail.com
}

\section{RESUME}

Les pays de l'Afrique subsaharienne sont fortement affectés par les affres des changements climatiques. La présente étude vise à analyser les perceptions paysannes sur la variabilité pluviométrique, les mesures adaptatives dans le moyen Bénin. Des informations ethno-climatiques collectées aux moyens d'une enquête par questionnaire sur un échantillon de 270 acteurs, ont permis d'apprécier les stratégies et les mesures d'adaptation mises en œuvre par les paysans. Le calcul de l'indice pluviométrique et la détermination des séquences sèches et humides sur la série pluviométrique de 1941 à 2010, ont permis de mieux étudier la variabilité pluviométrique. Les résultats des analyses ont montré que les principales mutations pluviométriques observées selon les perceptions paysannes sont le démarrage tardif, l'arrêt précoce et l'irrégularité des pluies (respectivement 96, 94 et $83 \%$ de réponses positives). Les conséquences se traduisent par la baisse de la productivité et du rendement agricole, le bouleversement et la non maîtrise du calendrier agricole. Les stratégies d'adaptation sont principalement l'extension des espaces agricoles, la reconversion socioprofessionnelle vers des secteurs d'exploitation forestière comme la production et la commercialisation du bois et du charbon de bois, contribuant ainsi à la régression de la couverture végétale de la région. Des mesures d'accompagnement (formation, encadrement, financement) méritent d'être prises pour identifier et vulgariser les mesures d'adaptation durables aux changements climatiques au Centre du Bénin.

(C) 2019 International Formulae Group. All rights reserved.

Mots clés: Centre du Bénin, mutations pluviométriques, perceptions, stratégies d'adaptations paysannes, destruction de la couverture végétale.

\section{Perceptions and farmers adaptation strategies to rainfall variability in the centre of Benin}

\begin{abstract}
Sub-Saharan Africa countries are strongly affected by climate change throes. The present study aims at analysing the farmers' perceptions on rainfall variability, the adaptation strategies in the middle Benin. Ethnoclimatic information collected, using questionnaire on a sample of 270 actors was used to appreciate the
\end{abstract}


adaptation strategies developed by farmers. The calculation of the rainfall index and the determination of the wet and dry sequences on the rainfall series of 1941 to 2010 , were used to better study the rainfall variability. The rainfall mutations observed according to farmers perceptions are the delay in the beginning, sudden stop and irregularity of rains $(96,94$ and $83 \%$ of positives responses respectively). Consequences are the reduction of the production and agricultural yield and the perturbations of the agricultural calendar. The adaptation strategies are mainly extension of farmlands, socio-professional reconversion towards forest exploitation sectors such as the production and the commerce of wood and wood charcoal, so contributing to the depletion of the vegetation cover of the region. Sustainable adaptation strategies to climate change should be identified and disseminated in the Centre of Benin through follow up measures such as training, monitoring and funding. (C) 2019 International Formulae Group. All rights reserved.

Keywords: Centre of Benin, rainfall mutations, perceptions, farmers adaptation strategies, depletion of vegetation cover.

\section{INTRODUCTION}

Les activités socioéconomiques sont fortement influencées par les facteurs climatiques en Afrique subsaharienne plus que partout ailleurs. Or, les dernières décennies de la fin du deuxième millénaire ont été marquées par une évolution rapide des climats à l'échelle globale (Boko et al., 2007; Odjugo, 2011; Puget et al., 2010) et une forte occurrence des événements pluviométriques extrêmes (IPCC, 2007).

En Afrique de l'ouest, cette évolution est marquée entre autres, par une variabilité pluviométrique sans précédent, du reste depuis le siècle passé. Ainsi, la plupart des travaux ont montré que les totaux pluviométriques annuels des décennies 1970 et 1980 sont caractérisés par des baisses sensibles, et la survenance d'années particulièrement pluvieuses avec des retombées socio-écologiques parfois considérables. Les travaux de Ogouwalé (2006), Yabi et al. (2011), ont montré que le Bénin est aussi sujet à une forte variabilité pluviométrique. Celle-ci se manifeste, en particulier, par une modification du régime des précipitations et par une diminution des hauteurs annuelles.

Dans un contexte d'agriculture essentiellement pluviale, les calendriers agricoles sont calés sur les rythmes des événements pluvieux. Un tel contexte pluviométrique a tôt fait de perturber les cycles culturaux, de bouleverser le calendrier agricole traditionnel et de rendre non opérationnelles les normes culturales en vigueur chez les populations paysannes (Ogouwale, 2001). Ainsi, la vulnérabilité des activités agricoles se manifeste par une détérioration des rendements et des pertes importantes de récoltes (Ogouwalé, 2006; Issa, 2012). Suivant les types d'activités et en se référant aux conditions pluviométriques "anciennes" ou "normales" qui fondent les calendriers habituels de déroulement des activités, les paysans perçoivent également les modifications pluviométriques saisonnières et annuelles (Yabi et Afouda, 2007; Yabi et Boko, 2008; Ouédraogo et al., 2010, Chabi et al., 2018).

Cette étude se base sur les fondamentaux de la perception et de l'approche par les moyens d'existence durables car les pratiques des agriculteurs, les choix techniques qu'ils font et les changements qui y sont liés découlent de leur conception des choses (Agossou et al., 2012; Ruault, 2007). La perception peut être définie comme l'action de saisir, de comprendre, de se représenter ou d'interpréter des phénomènes ou réalités par les sens et/ou par l'esprit (Kabré, 2008 cité par Ouoba, 2013). Dans le cadre de cette recherche, les perceptions se réfèrent aux mutations pluviométriques ressenties ou observées par les paysans. Elles se rapportent également aux effets desdites manifestations sur les activités selon les connaissances paysannes. 
Deux types de perceptions sont généralement considérés. Il s'agit de la perception collective et de la perception individuelle (Aho et al., 2006 ; Agossou et al., 2012). La perception collective est celle des populations locales et concerne les manifestations physiques et les effets ressentis par l'ensemble des producteurs de façon générale. Les perceptions individuelles quant à elles, sont exprimées par chaque producteur ou groupe de producteurs qui vivent dans des conditions socio-économiques similaires et qui appartiennent à un même réseau social ou des producteurs qui ont leurs exploitations dans une unité de paysage donnée (Ouoba et al., 2018; Atidegla et al., 2017). Selon Yegbemey et al. (2014), les perceptions individuelles peuvent être psychiques ou sensorielles. Elles sont psychiques, lorsqu'elles dépendent des facteurs fonctionnels tels que les expériences, les notions de valeurs, les attentes, les besoins, les opinions et les normes socioculturelles. Elles sont sensorielles quand elles dépendent des facteurs structurels qui ne sont rien d'autres que les cinq sens (la vue, l'ouïe, l'odorat, le toucher et le goût). Dans cette recherche, l'accent est beaucoup mis sur les perceptions individuelles psychiques des acteurs répondants. Par essence, qu'elles soient collectives ou individuelles, les perceptions sont subjectives (Leeuwis et Van den Band, 2003) dans la mesure où elles dépendent des connaissances locales et des savoirs empiriques sans oublier les croyances (Brou et al., 2005). Les perceptions impliquent le comportement, les différentes pratiques, les différentes actions/réactions, les raisons explicites développées ou utilisées par les producteurs face aux différentes modifications climatiques ressenties. Ces perceptions induisent donc des mesures développées pour résoudre un problème ou pour en limiter les effets. La connaissance et la prise en compte de ces perceptions est donc nécessaire car elles déterminent les mesures d'adaptions chez les paysans (Agossou et al., 2012 ; Ouédraogo et al., 2010), lesquelles mesures engendrent parfois des effets négatifs sur les composantes environnementales notamment le couvert végétal. En effet, des mesures endogènes ou non d'adaptions sont adoptées par les paysans pour réduire les effets néfastes des mutations pluviométriques. Ces mesures concernent surtout les modifications apportées aux systèmes culturaux, la reconversion socioprofessionnelle (Issa, 2012; Ogouwalé, 2006). Concernant la reconversion socioprofessionnelle, elle concerne la ruée vers l'exploitation forestière contribuant du coup à la régression des formations végétales. Par exemple, la FAO (2000) avait conclu qu'une perte d'environ 70000 ha serait enregistrée dans la période allant de 1990 à 2000 au Bénin. Ces différentes statistiques convergent sur le fait qu'il y a une régression inquiétante des surfaces forestières au Bénin. Le centre du pays est caractérisé par des perturbations enregistrées dans l'occupation et l'utilisation des terres, avec une pression de plus en plus forte sur les ressources marquée çà et là par des mouvements de populations (Oloukoi, 2013; Orekan, 2007).

Le présent travail analyse les perceptions paysannes sur la variabilité pluviométrique et les différentes mesures adaptatives dans le moyen Bénin (Figure 1). Il est initié pour compléter les résultats d'une thèse sur les mutations spatiotemporelles de l'occupation du sol au centre du Bénin (Oloukoï, 2012). En effet, au cours des investigations sur les déterminants de la dynamique de l'occupation du sol, la plupart des paysans répondants ont considéré que les aléas climatiques (pluviométriques notamment) figurent parmi les déterminants de la dynamique agraire et par ricochet de la dynamique de l'occupation du sol. Ainsi cette recherche est partie d'une hypothèse de base selon laquelle l'utilisation des terres à des fins agricoles est influencée par la manière dont les paysans perçoivent les mutations observées dans la pluviométrie ces dernières décennies. Autrement dit, cette recherche part $\mathrm{du}$ fait que les perceptions paysannes qu'elles soient subjectives ou objectives, induisent des 
adaptations susceptibles d'aggraver la dégradation des composantes environnementales (végétation, sol, eaux) du milieu. Il est donc nécessaire de mieux appréhender ces perceptions paysannes relatives aux mutations pluviométriques et les mesures adaptatives qui en découlent. Les résultats ajoutés aux autres connaissances pourraient orienter les politiques globales ou régionales de développement rural durable.

\section{MATERIEL ET METHODES}

\section{Cadre géographique de l'étude}

Le milieu d'étude est compris entre $1^{\circ} 38^{\prime}$ et $2^{\circ} 46^{\prime}$ de longitude Est d'une part et entre $7^{\circ} 30^{\prime}$ ' et $9^{\circ} 00^{\prime}$ de latitude Nord d'autre part (Figure 1). La population est constituée de groupes sociocommunautaires autochtones (Nago, Mahi, Bariba, Peulh) et immigrés encore appelés colons agricoles (Fon, Yom, Yoa-Lokpa, Gua, Adja, Dendi et apparentés).

Estimé à environ 253.052 habitants en 1979 (RGPH I), l'effectif de la population du secteur d'étude est passé respectivement à 390.061 habitants en 1992 (RGPH 2) et à 634.838 habitants en 2002 (RGPH 3). Ainsi cette population a connu un taux d'accroissement annuel de 3,5\% entre 1979 et 1992 contre $3,04 \%$ pour tout le territoire national. Entre 1992 et 2002, ce taux est passé à $4,6 \%$ contre $3,23 \%$ pour tout le pays (INSAE, 2003). La population est agricole (l'activité principale concerne l'exploitation de la terre à des fins de production végétale et/ou animale) à plus de $72 \%$ avec un nombre de ménages agricoles estimé à 78.105 sur un total de 108.384 ménages du secteur d'étude (Tableau 1).

Dans l'ensemble, la densité moyenne de la population du milieu est encore faible (51 habitants/km2) par comparaison à la densité moyenne nationale $(87,51$ habitants/km2), ce qui montre la disponibilité des terres agricoles. Les communes de Glazoué, Dassa-Zoumè et Savalou ont les plus fortes valeurs de densité (respectivement 70 , 66 et 54 habitants $/ \mathrm{km} 2$ ) alors que les communes de Savè, Bantè et Ouèssè sont les faiblement peuplées (39, 40 et 49 habitants $/ \mathrm{km} 2$ respectivement). En dehors des chefs-lieux de communes qui sont urbains, le reste du milieu est rural.

Le paysage végétal est constitué d'une gamme de formations allant des forêts denses aux mosaïques de cultures. Il est dominé par la savane arborée et arbustive (plus de 50\%) suivi des champs et jachères et de la forêt claire et savane boisée. Quant aux sols, ils sont essentiellement ferrugineux (en dehors des vallées où il $\mathrm{y}$ a des enclos de sols ferralitiques et hydromorphes), lessivés plus ou moins concrétionnés, difficiles à travailler, exposés à l'érosion et d'aptitude culturale moyenne.

Le Centre du Bénin se situe dans la zone de transition entre le climat subéquatorial du Sud et le climat soudanien du Nord (Boko, 1988; Afouda, 1990). Le régime pluviométrique est à cheval sur celui de la distribution bimodale du Sud et celui de la distribution unimodale du Nord. Les isohyètes sont distribuées de façon concentrique, délimitant des poches plus sèches (autour de $1000 \mathrm{~mm}$ à Toui et à Aklampa), et des poches plus humides (1200 $\mathrm{mm}$ à Dassa-Zoumé) comme le montre la Figure 2.

Les activités rurales concernent essentiellement la production végétale qui occupe les ménages agricoles. Les principales productions sont vivrières et concernent les cultures de maïs (Zea mays) (Adimi et al., 2018), du manioc (Manihot esculenta), de l'igname (Dioscorea alata), de l'arachide (Arachis hypogaea), du niébé (Vigna unguiculata) sans oublier le riz (Oryzea sativa) cultivé dans les bas-fonds notamment à Glazoué, Dassa-Zoumè, Savalou et Ouèssè). Aux cultures vivrières s'ajoutent le coton (Gossypium $s p$ ), principale culture d'exportation encouragée par l'Etat et l'anacardier (Anacardium occidentale) cultivé suivant un système agro-forestier au cours des premières années. Il s'agit d'une production pluviale de type familial sur des superficies modestes (dépassant rarement $1 \mathrm{ha}$ ). 
S'agissant de la production animale, elle concerne l'élevage des ruminants, notamment des bovins. Cette activité qui est de type extensif est menée par les communautés peulhs. On distingue les peulhs éleveurs qui ont installé des campements et qui se sont sédentarisés dans le milieu, et les peulhs transhumants en provenance du nordBénin ou des pays limitrophes (Nigéria, Niger, Burkina) dont le séjour dans le milieu est saisonnier. Dans tous les cas, le pâturage et les points d'eau naturels, constituent les sources quasi-exclusives d'alimentation du bétail.

Au total, les activités rurales du centre Bénin dépendent étroitement de la répartition spatiotemporelle des pluies. Dans un tel contexte, les communautés paysannes sont très vulnérables à la variabilité pluviométrique.

\section{Méthodes \\ Collecte des données}

Les données climatiques utilisées concernent les hauteurs pluviométriques et les valeurs de l'évapotranspiration potentielle (ETP) des stations de Savè (représentative du Sud) et de Parakou (représentative du Nord et plus proche de la commune de Tchaourou). Elles se rapportent à la période allant de 1941 à 2010 et ont été extraites du fichier de l'Agence pour la Sécurité de la Navigation Aérienne en Afrique et à Madagascar (ASECNA-Bénin).

A ces données s'ajoutent des informations ethno-climatiques (perceptions paysannes de variabilité pluviométrique, les incidences de cette variabilité sur les activités rurales) et sur les stratégies d'adaptation mises en œuvre. Elles ont été obtenues auprès des différentes catégories socioprofessionnelles concernées par la problématique étudiée grâce à une enquête au moyen de questionnaire. Ainsi, les agriculteurs, les agro-éleveurs et les personnes ressources (agents d'encadrement rural, agents d'ONG intervenant dans le domaine de l'agriculture) ont été pris en compte (Tableau 2). Ici le mot "agro- éleveurs" désigne les peulhs éleveurs de gros bétail sédentarisés dans le milieu qui combinent la production végétale avec l'élevage du gros bétail. Il s'agit des éleveurs qui, pendant la saison pluvieuse s'adonnent à la production végétale vivrière (mil, sorgho maïs, igname) voire rentière (coton) pour compléter leur activité principale qui est l'élevage du gros bétail. Pendant la saison sèche où les ressources pâturées se raréfient, ils s'occupent du troupeau qui a besoin d'être conduit vers les contrées plus ou moins éloignées à la recherche de pâturage et de points d'abreuvement. Quant au mot "'agriculteurs" il désigne dans ce cadre les paysans qui utilisent la terre à des fins exclusives de production végétale. Autrement dit, il s'agit des paysans qui s'adonnent uniquement à la production végétale. En phase avec l'objectif de la recherche, les questions posées sont relatives aux perturbations climatiques (tendance des totaux pluviométriques annuels, démarrage et fins des saisons, poches de sécheresse au cœur de la saison pluvieuse), la période de référence des perturbations et les mesures d'adaptation à savoir l'ajustement des pratiques agricoles, la reconversion professionnelle. Un accent particulier a été mis sur les adaptations à forte incidence sur l'occupation du sol (extension des superficies agricoles, valorisation des basfonds et plaines d'inondation, exploitation forestière, transhumance). A chaque question, plusieurs options de réponses sont proposées au répondant et un code $(1,2,3, \ldots)$ est attribué à chaque réponse pour faciliter le dépouillement. La taille de l'échantillon est arbitrairement fixée à 240 chefs de ménages pour les paysans au regard des ressources financières et humaines limitées. Les 240 chefs de ménages sont constitués de 160 agriculteurs et 80 agro-éleveurs. Les 240 paysans ont été répartis équitablement dans 8 Communes (soient 30 par Commune) sans tenir compte de leur poids démographique vu la taille réduite de l'échantillon. Les communes considérées dans l'échantillonnage sont Glazoué, Dassa-Zoumè, Savalou, Savè, 
Ouèssè, Tchaourou et Bassila. Les deux autres communes à savoir Kétou et Djidja se situent dans la lisière sud et ne sont concernées que de façon marginale car c'est seulement une petite partie de leur espace qui fait partie de la zone d'étude. Dans chaque Commune, le choix des paysans a été fait avec le concours des agents des Secteurs Communaux de Développement Agricole (SCDA) sur la base des capacités productives des localités et des personnes (Tableau 2). Le questionnaire est administré à tous les 240 paysans au cours des entretiens individuels. Ces entretiens individuels ont été complétés par des observations directes dans des exploitations agricoles, les parcours de pâturages, les entrepôts de madriers et charbons de bois. Elles ont permis de compléter les informations obtenues par questionnaire et de constater les mesures d'adaptation et leurs incidences sur l'occupation et l'utilisation du sol. En plus de ces acteurs principaux, l'échantillon a pris en compte 30 personnes ressources dont 11 agents d'encadrement rural et 19 agents d'ONGs intéressées par le développement rural et les problèmes environnementaux. Les questions adressées à ces acteurs ont porté essentiellement sur les mutations pluviométriques perçues en vue de déceler les aspects de convergence et de divergence éventuelles avec les perceptions paysannes.

Les investigations de terrain se sont déroulées au cours de la période allant de novembre 2012 à janvier 2013. Le choix de cette période tient au fait qu'elle correspond à la saison sèche où les paysans sont plus disponibles d'une part et au moment où les pistes d'accès aux différentes localités et exploitations ainsi que les autres sites d'observation, sont plus praticables d'autre part.

Le calcul de l'indice pluviométrique (IP) a permis de mieux étudier la variabilité pluviométrique et de mettre en évidence les années sèches et les années humides. Encore appelé indice centré réduit, ou indice standardisé des précipitations, il est obtenu en faisant le rapport entre l'écart à la moyenne et l'écart-type des hauteurs pluviométriques annuelles. Il s'écrit de la manière suivante :

$\boldsymbol{I P}=(\boldsymbol{x} \boldsymbol{i}-\boldsymbol{x}) / \boldsymbol{\sigma}$

Dans cette formule, $\mathrm{x}$ correspond à la hauteur moyenne de précipitations, xi à la hauteur de pluie d'une année i et $\sigma$ représente la déviation standard (écart-type). Les valeurs positives traduisent des excédents pluviométriques tandis que les valeurs négatives indiquent les déficits pluviométriques. Le choix de cet indice tient au fait qu'il est recommandé par l'Organisation Météorologique Mondiale (OMM, 2012). Il se calcule aisément et ne nécessite pas l'utilisation de logiciel spécifique. Par ailleurs son utilisation est approuvée par plusieurs auteurs (Bodian et al., 2011; Kouassi et al., 2010 ; Sarr, 2008) dans différentes régions d'Afrique de l'Ouest.

La détermination des séquences pluviométriques sèches et humides s'est inspirée des travaux de Sané et al. (2008) et de Zachari et al. (2012). Ainsi une séquence est considérée comme sèche lorsque pendant la saison agricole, il s'écoule au moins 10 jours sans pluie (faux départ de pluie si la séquence intervient juste après le démarrage de la saison). Un tel contexte est capable d'affecter négativement les besoins en eau des plantes à racines superficielles comme les cultures saisonnières. Par contre une séquence est considérée comme humide lorsque pendant la saison agricole et au cours de la même semaine trois événements pluvieux d'au moins $30 \mathrm{~mm}$ s'enregistrent ou lorsque la pluviométrie hebdomadaire est supérieure ou égale à $100 \mathrm{~mm}$. Dans ces conditions, les exploitations agricoles sont susceptibles d'être inondées. En outre, l'utilisation de la fréquence de démarrage tardif (lorsqu'il intervient 2 décades au moins après la date moyenne) et de fin précoce (lorsqu'elle intervient 2 décades au moins avant la date moyenne) des pluies, a permis de mieux vérifier les perceptions paysannes. 


\section{Analyse statistique}

Les outils de la statistique descriptive (fréquence relative, pourcentage, analyse factorielle des correspondances) complétés par le test de concordance de Mann Kendall ont été utilisés pour traiter les données relatives aux perceptions paysannes sur la variabilité pluviométrique et les mesures d'adaptation utilisées. La fréquence relative (F) est obtenue par la formule suivante : $\mathrm{F}=$ $\mathrm{f} / \mathrm{N}$

$\mathrm{f}=$ fréquence absolue ou effectif de répondants concernés par une perception ou opinion ; $\mathrm{N}=$ effectif total des répondants. $\mathrm{F}$ est multiplié par cent pour obtenir des pourcentages. $\mathrm{F}=(\mathrm{f} / \mathrm{N}) \times 100$

L'utilisation de la fréquence et du pourcentage a permis de résumer les réponses aux questions et de faire des comparaisons de valeurs.

S'agissant de l'analyse factorielle des correspondances, elle a pour but de décrire les relations entre les modalités de deux ensembles de caractères. Ici, il s'agit des groupes socio-professionnels et mesures d'adaptation organisés dans un tableau de contingence. Cette analyse a permis de faire une lecture croisée entre les différents groupes socio-professionnels et les mesures adaptatives mises en œuvre face aux effets des aléas pluviométriques perçus.

En ce qui concerne le test de concordance de Mann Kendall, il est effectué au seuil de significativité de $5 \%$ à l'aide du logiciel SPSS v.16. Il s'agit d'un test non paramétrique qui a permis de vérifier statistiquement la significativité du classement par ordre d'importance (ampleur et fréquence) des 5 épisodes pluviométriques les plus perçus par les paysans répondants ayant réussi à faire le classement (180).

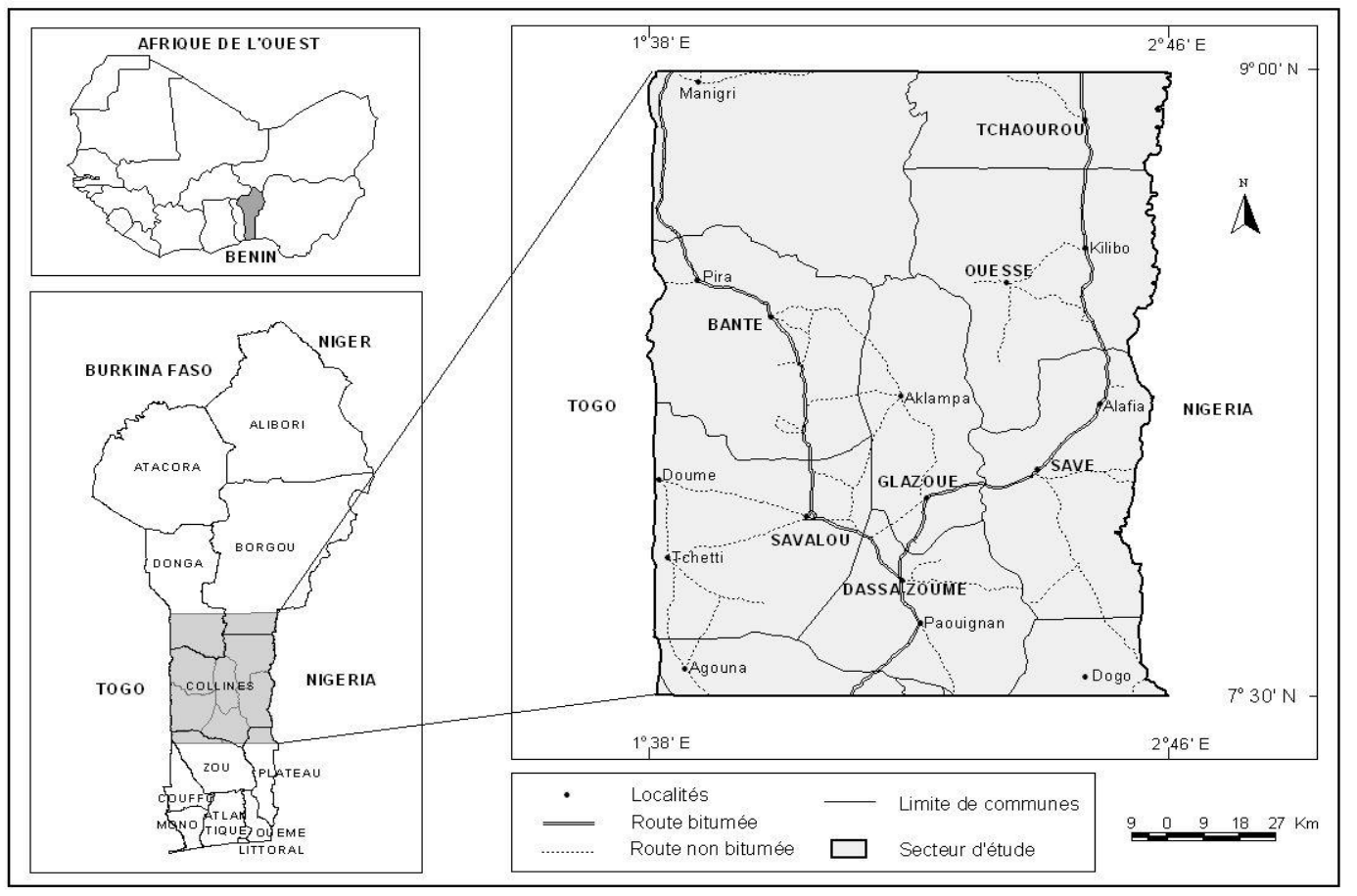

Figure 1: Secteur d'étude. 
Tableau 1: Population du milieu d'étude en 2010 et importance agricole.

\begin{tabular}{lcccccc}
\hline \multicolumn{1}{c}{ Communes } & $\begin{array}{c}\text { Nombre de } \\
\text { Ménages }\end{array}$ & $\begin{array}{c}\text { Nombre de Ménages } \\
\text { agricoles }\end{array}$ & \multicolumn{2}{c}{ Population } & \multicolumn{2}{c}{$\begin{array}{c}\text { Population } \\
\text { agricole }\end{array}$} \\
\cline { 4 - 7 } & & 3881 & 21797 & 22586 & 44383 & 28537 \\
\hline Tchaourou (Sud) & 6632 & 2406 & 9483 & 10101 & 19584 & 14193 \\
Kétou (Nord) & 3407 & 5452 & 29164 & 29396 & 58559 & 46900 \\
Bassila (Sud) & 7557 & 2267 & 7342 & 7619 & 14961 & 13854 \\
Djidja (Nord) & 2566 & 12406 & 55788 & 58372 & 114159 & 89419 \\
Bantè & 17689 & 17981 & 62814 & 67800 & 130614 & 106007 \\
Dassa-Zoumè & 24151 & 17517 & 60546 & 65215 & 125760 & 105664 \\
Glazoué & 22458 & 17057 & 67109 & 67512 & 134622 & 119220 \\
Ouèssè & 20516 & 18894 & 69727 & 75875 & 145601 & 102435 \\
Savalou & 29430 & 10706 & 47202 & 46981 & 94182 & 68042 \\
Savè & 16246 & 108566 & 430970 & 451455 & 882425 & 694270 \\
\hline Total & 150654 & & & & \\
\hline
\end{tabular}

Source: Projection faite sur 2010 sur la base des données du RGPH 2 (1992) et RGPH 3 (2002).

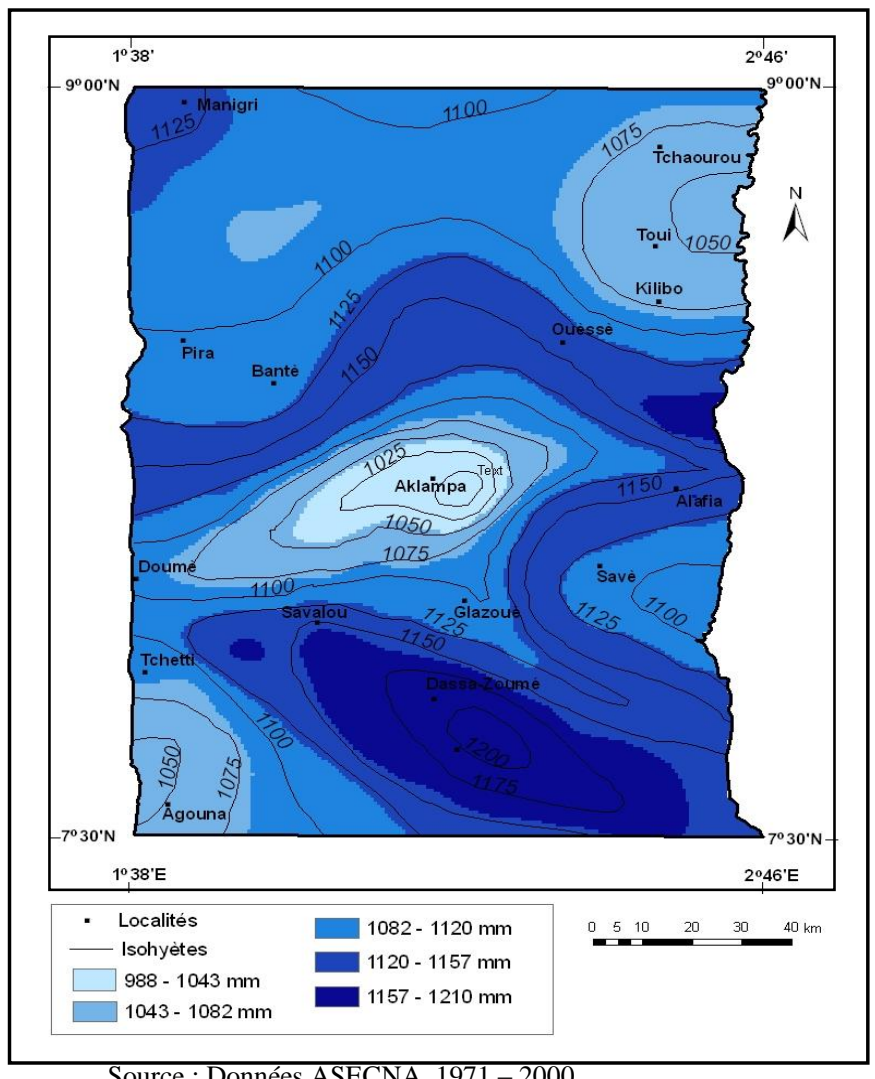

Source : Données ASECNA, $1971-2000$.

Figure 2: Répartition spatiale des totaux pluviométriques dans le Centre du Bénin (1971-2000). 
Tableau 2: Echantillon des personnes enquêtées.

\begin{tabular}{ccl}
\hline $\begin{array}{c}\text { Catégories socio- } \\
\text { professionnelles }\end{array}$ & Effectif & \multicolumn{1}{c}{ Observations } \\
\hline Agriculteurs & 160 & $\begin{array}{l}\text { 20 producteurs agricoles ont été choisis par commune à raison de 10 } \\
\text { jeunes (âge compris entre 18 et 45 ans) et 10 vieux (âge supérieur à } \\
45 \text { ans) } \\
10 \text { producteurs agro-éleveurs ont été choisis par commune à raison } \\
\text { de } 5 \text { jeunes (âge compris entre 18 et 45 ans) et 5 vieux (âge supérieur } \\
\text { à } 45 \text { ans) } \\
6 \text { responsables de production végétale, } 5 \text { responsables de production } \\
\text { animale et 19 agents d'ONG }\end{array}$ \\
Agro-éleveurs & 80 \\
Personnes & 30 & \\
\hline ressources
\end{tabular}

\section{RESULTATS}

Les résultats sont axés sur les perceptions paysannes sur la variation pluviométrique, l'évidence scientifique de la variation pluviométrique, les stratégies d'adaptation des acteurs aux conséquences de cette variation et enfin les implications des changements sur le couvert végétal.

\section{Perceptions paysannes de la variabilité pluviométrique}

Les paysans interrogés perçoivent les perturbations pluviométriques sous plusieurs aspects en fonction de leur connaissance (Figure 3).

La quasi-totalité des paysans répondants ont évoqué le démarrage tardif, l'arrêt précoce et l'irrégularité des pluies (respectivement 96, 94 et $83 \%$ de réponses positives). Ensuite, les faux départs pluviométriques, une tendance à la baisse des pluies et les excès momentanés des pluies sont cités comme des aspects des mutations pluviométriques par les acteurs. Quelques-uns (22\%) ont tout de même évoqué une tendance à la hausse des pluies. Quelques déclarations ont permis de catégoriser les mutations pluviométriques perçues par les paysans (Tableau 3).

Les paysans font référence aux évènements historiques (années ou période de naissance ou de décès des connaissances), politiques (années des indépendances, période révolutionnaire, règne de tel ou tel président) et sociaux (graves crises alimentaires) pour les moments où les mutations ont commencé par être perçues. Ainsi, selon $65 \%$ des vieux, la baisse des totaux pluviométriques, le démarrage tardif, la fin précoce et l'irrégularité des pluies ont commencé depuis les années 1970 (période révolutionnaire) alors que les excès momentanés des pluies sont devenus plus récurrents depuis les années 1990 (période où le Président Kérékou a quitté le pouvoir), ce qui se solde par des inondations récurrentes.

La majorité des personnes ressources (11/11 pour les agents d'encadrement et 16/19 pour les agents d'ONGs) interrogées ont confirmé l'instabilité qui caractérise les pluies. Ces personnes ressources ont surtout mis l'accent sur les démarrages tardifs, les fins précoces et les séquences sèches au cœur des saisons. Leurs avis sont par contre partagés sur la tendance générale à la hausse ou la baisse des pluies. Ainsi, 5/11 des agents d'encadrement et $8 / 19$ pour des agents d'ONGs pensent que les pluies ont tendance à baisser, alors que $3 / 11$ des agents d'encadrement et $6 / 19$ des agents d'ONGs pensent plutôt que les pluies ont tendance à augmenter. Pour le reste, il est difficile voire impossible d'identifier une tendance, vu le caractère très irrégulier des pluies.

Chaque paysan répondant a cité au moins 5 épisodes comme indicateurs des 
mutations pluviométriques. La synthèse des classifications (suivant l'ampleur et la fréquence) faites par les acteurs interrogés (Tableau 4) confirme la prééminence du démarrage tardif et de l'arrêt précoce des pluies. Par contre la baisse et la hausse des pluies ne font pas partie des 5 premiers épisodes pluviométriques cités. Il importe de noter qu'il n'y a eu que 180 paysans répondants qui ont pu classer par ordre décroissant les 5 premiers épisodes pluviométriques (suivant l'ampleur et la fréquence) perçus. Selon les autres paysans, il est impossible de faire un classement dans la mesure où les différents aléas surviennent suivant des ampleurs et fréquence très variables.

Une perturbation du cycle pluviométrique affecte le système de culture comme l'expriment plus de $97 \%$ des acteurs ; ce qui a nécessité des stratégies locales d'adaptation. Les opinions exprimées par la population révèlent que les cinq dernières années (2004-2009) ont été plus sèches tandis que les dix et quinze dernières années (19992009 ; 1994-2009) ont été normales. Selon les mêmes perceptions, les vingt dernières années (1989-2009) ont été plus pluvieuses que les dix et quinze dernières (Tableau 5).

Les conséquences de cette variation pluviométrique selon les perceptions paysannes se traduisent par la baisse de la productivité et du rendement agricole, le bouleversement du calendrier agricole, l'échec des semis. Selon les enquêtes, la baisse de productivité et de rendement agricole s'apprécie chez les producteurs par des comparaisons empiriques (en se référant à la taille du grenier, au nombre de sacs ou de bassines) des quantités de récoltes. Ils pensent qu'avec les mêmes dimensions de superficies culturales et avec des efforts similaires, les quantités de récoltes baissent en rapport avec les irrégularités pluviométriques. De même, les dates de semis (et partant les autres activités culturales) sont devenues incertaines selon les paysans dans la mesure où le démarrage et la fin des saisons sont erratiques sans oublier les poches de sècheresse fréquentes au cœur de la saison agricole. L'échec des semis est également lié à l'instabilité des pluies qui induit la pourriture des semences ou des jeunes pousses de cultures selon les producteurs.

Les stratégies d'adaptation les plus remarquables sont la reconversion dans d'autres secteurs d'activités (exploitation forestière, artisanat, élevage, pèche, production du charbon de bois etc.), la migration de certains acteurs vers d'autres régions, le déplacement du front des activités agricoles vers les bas-fonds ou zones humides, l'attente de la pluie après imploration des dieux, l'adoption des semences de variétés à cycle court.

Il convient de vérifier les perceptions paysannes en utilisant les données pluviométriques. A cet effet l'évolution des indices pluviométriques et l'occurrence des séquences pluvieuses sèches ou humides ont été analysées.

\section{Variabilité pluviométrique dans le centre du Bénin}

L'analyse des anomalies pluviométriques des stations de Savè et de Parakou (Figure 4) montre que le centre du Bénin a connu une alternance d'années très pluvieuses, normales (moyennes) et déficitaires sans aucune périodicité apparente. La Figure 4 montre que la région a toujours connu des années de déficits ou d'excédents pluviométriques mais l'occurrence des années déficitaires est plus élevée au cours des années 1970 et 1980 (environ une année sur deux) tandis que les décennies 1940, 1950 et 1960 sont caractérisées par une fréquence plus accrue des années excédentaires.

La forte occurrence des années déficitaires au cours des années 1970 et 1980 correspond aux perceptions paysannes des mutations pluviométriques. Les graves crises alimentaires engendrées par les sécheresses de ces années et qui ont marqué la mémoire des 
personnes âgés justifient certainement cette concordance des perceptions avec les réalités pluviométriques du milieu. Mais ils ne font pas référence aux années 1940, 1950 et 1960 pour situer les fortes pluies. La plupart des vieux paysans étant analphabètes (85\%), ils ne disposent pas d'archives pouvant leur permettre de se rappeler des conditions pluviométriques de ces années.

Une étude comparée et détaillée des perceptions paysannes subjectives du Tableau 3 avec les données pluviométriques montrent les observations suivantes pour chaque « type de mutation $»$ :

- Par rapport aux démarrages tardifs des pluies, les analyses ont montré qu'au cours des trente dernières années (19812010), 4 années sur 10 et 3 années sur 10 ont été affectées par ce phénomène respectivement à Savè et à Parakou.

- S'agissant des arrêts précoces, ils ont concerné 4 années sur 10 en moyenne pour les deux stations. Toutefois, il faut préciser que la fréquence des années simultanément affectées par les deux phénomènes n'est que de 2 années sur 10.

- Quant aux faux départs pluviométriques, leur fréquence moyenne est de 4 années sur 10 pour les deux stations.

- En ce qui concerne les forts abats pluviométriques (excès momentanés des pluies) qui engendrent les inondations, ils concernent surtout les mois d'août et de septembre dont la fréquence des excédents est 5 et 6 années sur 10 respectivement à Savè et à Parakou au cours des 20 dernières années (19902010). Il convient de signaler que cette anomalie affecte surtout des années excédentaires et moyennes.

Un examen croisé des données montre que 8 et 7 années sur 10 (respectivement à Savè et à Parakou) sont concernées par au moins une des trois anomalies que sont le démarrage tardif, l'arrêt précoce et le faux départ. Ces anomalies affectent autant les années sèches que les années normales ou moyennes (dont la hauteur pluviométrique est comprise entre la hauteur moyenne de la série plus ou moins 20\%) et même les années excédentaires.

En définitive, les perceptions paysannes concernent plus les échelles saisonnières (début de la saison, cœur de la saison et fin de la saison) qui influencent beaucoup plus les activités (agricoles et pastorales) que les niveaux annuels. Mais, il se fait qu'une année considérée comme normale ou moyenne à l'échelle annuelle (généralement 6 années sur 10) connait souvent des perturbations mensuelles ou saisonnières qui impactent négativement les activités paysannes. De même l'année peut être excédentaire alors qu'elle a connu des perturbations au début et ou à la fin de la saison.

L'analyse des séquences sèches et pluvieuses (Figure 5) permet aussi de mieux comprendre la concordance entre les perceptions paysannes et la réalité pluviométrique du milieu. Partout, les séquences sèches sont plus fréquentes au début et à la fin de la saison agricole (mai, juin et octobre). La forte occurrence de ces séquences s'explique par les démarrages tardifs, les faux départs et l'arrêt précoce des pluies qui compromettent la production agricole et la disponibilité du pâturage. Ces constats sont concordants avec les perceptions paysannes relatives à la mauvaise répartition temporelle des pluies qui caractérise le climat du centre du Bénin.

$\mathrm{Au}$ final, les perceptions paysannes et les analyses scientifiques attestent que le centre du Bénin est sujet à une variabilité pluviométrique qui se manifeste entre autres par le démarrage tardif, la fin précoce des pluies sans oublier l'apparition des séquences sèches au début et en fin de la saison agricole de même que les séquences très pluvieuses au cœur de la saison. Ces différentes mutations pluviométriques affectent les activités agricoles et agro-pastorales des paysans. En 
réponses ces derniers adoptent des mesures adaptatives en fonction de leurs activités et de leurs connaissances empiriques.

En moyenne, deux campagnes agricoles sont mises en œuvre dans le centre du Bénin par an, les calendriers agricoles étant intimement calqués sur la répartition moyenne des pluies (Tableau 6). Ainsi, la première campagne commence en mars avec les premières pluies (bien que ce mois soit globalement sec), les producteurs commencent les travaux de préparation du sol (défrichement, labours, etc.). Les semis interviennent en avril-mai, quand le sol est suffisamment humide avec l'installation définitive des pluies. Les travaux d'entretien (sarclage, épandage d'engrais, traitement phytosanitaires) s'en suivent et les premières récoltes interviennent en juillet-août. Les travaux relatifs à la deuxième campagne quant à eux commencent en août où la préparation du sol et les semis interviennent. Les activités agricoles se poursuivent jusqu'aux récoltes qui interviennent en octobre-novembre.

S'agissant des activités pastorales, elles ont lieu durant toute l'année. Pendant la saison sèche, les ressources pâturées (fourrages et eau d'abreuvage) se raréfient, ce qui oblige les agro-éleveurs à migrer vers les régions moins austères (transhumance). En saison pluvieuse, les ressources pâturées sont disponibles à proximité des campements, ce qui permet aux agro-éleveurs de s'adonner aux activités de production agricole (végétale) en plus de l'activité pastorale.

Mais la réussite des deux campagnes agricoles annuelles est conditionnée par une bonne répartition des pluies nécessaire à la satisfaction des besoins en eau des plantes cultivées et naturelles (fourragères) et au rechargement des sources d'eau d'abreuvage des troupeaux. Dans ce contexte, les irrégularités saisonnières (début tardif, fin précoce, séquences sèches ou humides, insuffisances pluviométriques) compromettent sérieusement les activités paysannes et obligent les acteurs à adopter des mesures adaptatives.

\section{Adaptation à l'évolution pluviométrique dans le centre du Bénin}

L'adaptation se rapporte aux stratégies adoptées par les paysans (agriculteurs et agroéleveurs), dans le cadre de leurs activités, pour limiter les effets néfastes des mutations pluviométriques. Les enquêtes de terrain ont montré que face aux changements des précipitations, $92 \%$ des paysans interrogés adoptent des mesures adaptatives. Comme l'illustre la Figure 6, les types d'adaptation varient en fonction de la profession et de l'âge des paysans répondants. Ainsi l'axe 1 (qui prend en compte $65 \%$ de l'information) révèle que les mesures mises en œuvre par les agriculteurs diffèrent de celles adoptées par les agro-éleveurs. Chez les agriculteurs, les mesures utilisées concernent surtout l'extension des surfaces de culture, la mise en valeur des bas-fonds, l'adoption de nouvelles variétés culturales, l'exploitation forestière (fabrication du charbon et production du bois d'œuvre). Les agro-éleveurs quant à eux utilisent beaucoup plus l'émondage des arbres, l'allongement de la durée de transhumance et le déplacement des campements. Quant à l'axe $2(26 \%$ de l'information), il montre que les mesures souvent adoptées par les jeunes ne sont pas identiques à celles mises en œuvre par les vieux. Les jeunes agriculteurs ont souvent opté pour la reconversion professionnelle. Ainsi, ils s'intéressent à la fabrication du charbon et à la production du bois d'œuvre qu'ils considèrent comme des activités plus génératrices de revenus monétaires. A cela s'ajoute la diversification des activités qui permet aux jeunes d'associer aux travaux agricoles traditionnels d'autres activités comme le commerce informel de produits pétroliers, le maraichage, la conduite de taximoto. Quant aux agriculteurs âgés, ils ont plus opté pour la mise en valeur des basfonds, l'extension des superficies culturales. 
Les jeunes agro-éleveurs sont plus concernés par l'émondage massif des arbres et l'allongement de la durée de transhumance alors que leurs ainés utilisent souvent le déplacement des campements vers d'autres milieux pour s'adapter aux aléas pluviométriques.

Selon les investigations, les mesures d'adaptation utilisées visent à maintenir le niveau de la production végétale (extension de la surface cultivée, choix de nouvelles variétés culturales, exploitation des bas-fonds) et animale (déplacement des lieux de campement, émondage accrue des arbres, allongement de la durée de transhumance) et d'assurer des revenus alternatifs ou complémentaires (exploitation forestière, diversification des activités).

L'efficacité économique et environnementale de ces mesures reste à évaluer mais il y en a certaines qui affectent négativement le couvert végétal de la région. Il s'agit principalement de la mise en valeur des bas-fonds, l'émondage massif des arbres et l'exploitation forestière.

\section{Stratégies d'adaptation et destruction de l'environnement}

Les stratégies les plus adoptées par les producteurs agricoles sont principalement l'extension des espaces agricoles, la reconversion vers des secteurs d'exploitation forestière comme la production et la commercialisation du bois et du charbon de bois. S'il parait difficile de lier toutes ces stratégies aux seules conséquences de la variabilité pluviométrique, considérant aussi les stress économiques, sociaux, démographiques et culturels que subissent les populations, il importe toutefois de préciser que ces stratégies ont pris de l'ampleur depuis que l'agriculture a commencé à subir les conséquences des aléas. Beaucoup de ces agriculteurs ont opté aussi pour la reconversion vers d'autres secteurs d'activité, ou renforcent leurs activités agricoles par d'autres, notamment l'exploitation forestière.
Ainsi, de nouvelles activités autrefois reléguées au second rang, sont en train d'absorber un nombre important de pratiquants dans le Centre du Bénin. Il s'agit de l'exploitation et du commerce de bois et la production du charbon de bois (Figure 7). L'exploitation forestière par l'abattage de grands arbres pour la production du bois et du charbon de bois, est l'œuvre d'acteurs locaux et migrants avec parfois la complicité des autorités locales. Les investigations de terrain ont révélé que même les aires classées ou protégées ne sont pas épargnées par les exploitants à la recherche du bois.

En ce qui concerne la stratégie relative au déplacement du front des activités agricoles vers les bas-fonds, il faut aussi comprendre que la mise en valeur des basfonds n'est pas sans conséquence sur la biodiversité. Au-delà des atouts agronomiques et économiques dont ils disposent, les basfonds constituent des écosystèmes fragiles (Oloukoi et al., 2017). L'aménagement d'un bas-fond nécessite toujours un défrichement. Le défrichement accompagné des diverses techniques de nettoyage, affecte la biodiversité de ces écosystèmes. Les niches écologiques parmi lesquelles les terres humides sont les plus importantes, sont très souvent les seules sources de recolonisation végétale, mais cependant la menace sur leur disparition est grande. Au centre du Bénin, si le bas-fond a été pendant longtemps le lieu de prélèvement du bois de chauffe utilisé pour la fabrication du gari, de nos jours, les charbonniers ont décimé presque toutes les espèces ligneuses suite à la mise en culture de cet espace jadis marginalisé.

$\mathrm{Au}$ final, il importe de retenir qu'entre les perceptions locales et l'exploitation des ressources environnementales, il y a un lien ou un trait d'union 'incarné' par l'acteur-agriculteur ou éleveur transhumant lui-même, qui prélève des ressources de l'environnement (Djenontin, 2010; FAO, 2012). 


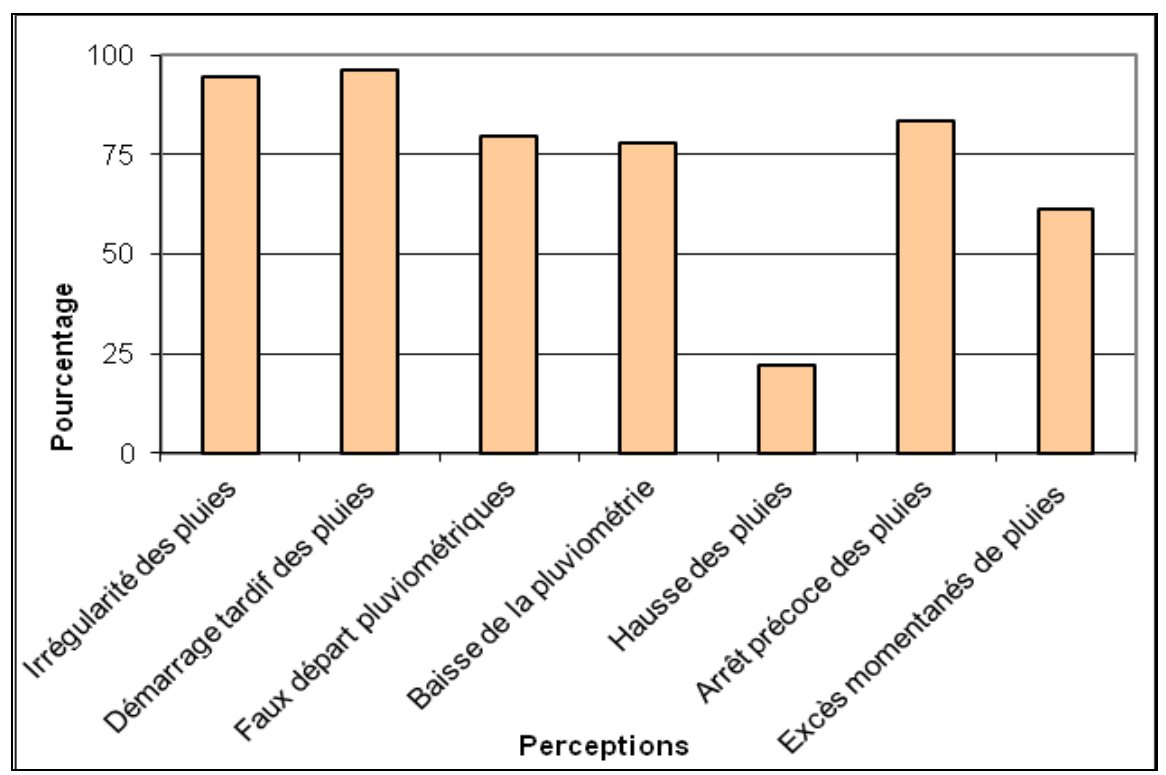

Figure 3: Principaux épisodes pluviométriques perçus par les paysans.

Tableau 3: Exemples des déclarations paysannes et mutations correspondantes.

\section{Déclarations paysannes}

Avant, la saison pluvieuse commençait en avril-mai, mais de nos jours, il faut attendre jusqu'en juin

Les récoltes de la deuxième saison sont devenues très incertaines car les pluies s'arrêtent brutalement avant la maturation des cultures

Les pluies commencent normalement, mais après les semis elles s'interrompent parfois jusqu'à un mois le temps que les jeunes poussent se dessèchent

Avant on arrive à déterminer les moments de démarrage, d'abondance et de l'arrêt des pluies, ce qui nous permet d'organiser les activités suivant un calendrier déterminé, mais actuellement, tout a changé

Alors que les pluies ont démarré en retard, elles tombent abondamment pendant une à deux semaines engendrant ainsi des inondations des exploitations

\section{Types de mutations}

Démarrage tardif des pluies

Fin précoce des pluies

Faux départs pluviométriques

Irrégularité des pluies

Excès momentanés des pluies ou abats pluviométriques

Tableau 4: Classification des épisodes cités.

\begin{tabular}{lcl}
\hline Episodes & Rang & Test de concordance de Mann Kendall \\
\hline Démarrage tardif des pluies & 1 & \\
Arrêt précoce des pluies & 2 & $\mathrm{~N}=180^{*}$ \\
Irrégularité des pluies & 3 & Alpha $=0,05$ \\
Faux départ pluviométriques & 4 & Probabilité $=0,0355$ \\
Excès momentanés de pluies & 5 & \\
\hline
\end{tabular}

*seules les réponses de 180 personnes sont exploitées dans cette analyse. 
Tableau 5: Opinions paysannes sur la pluviométrie des vingt dernières années.

\begin{tabular}{ccccc}
\hline \multirow{2}{*}{$\begin{array}{c}\text { Opinions des } \\
\text { acteurs }\end{array}$} & $\begin{array}{c}\mathbf{5} \text { dernières } \\
\text { années }\end{array}$ & $\begin{array}{c}\mathbf{1 0} \text { dernières } \\
\text { années }\end{array}$ & $\begin{array}{c}\mathbf{1 5} \text { dernières } \\
\text { années }\end{array}$ & $\begin{array}{c}\mathbf{2 0} \text { dernières } \\
\text { années }\end{array}$ \\
\hline Plus sèche & 67,38 & 15,60 & 11,43 & 5,71 \\
Normale & 24,11 & 68,09 & 55 & 35 \\
Plus pluvieuse & 8,51 & 16,31 & 33,57 & 59,29 \\
\hline Total & 100 & 100 & 100 & 100 \\
\hline
\end{tabular}

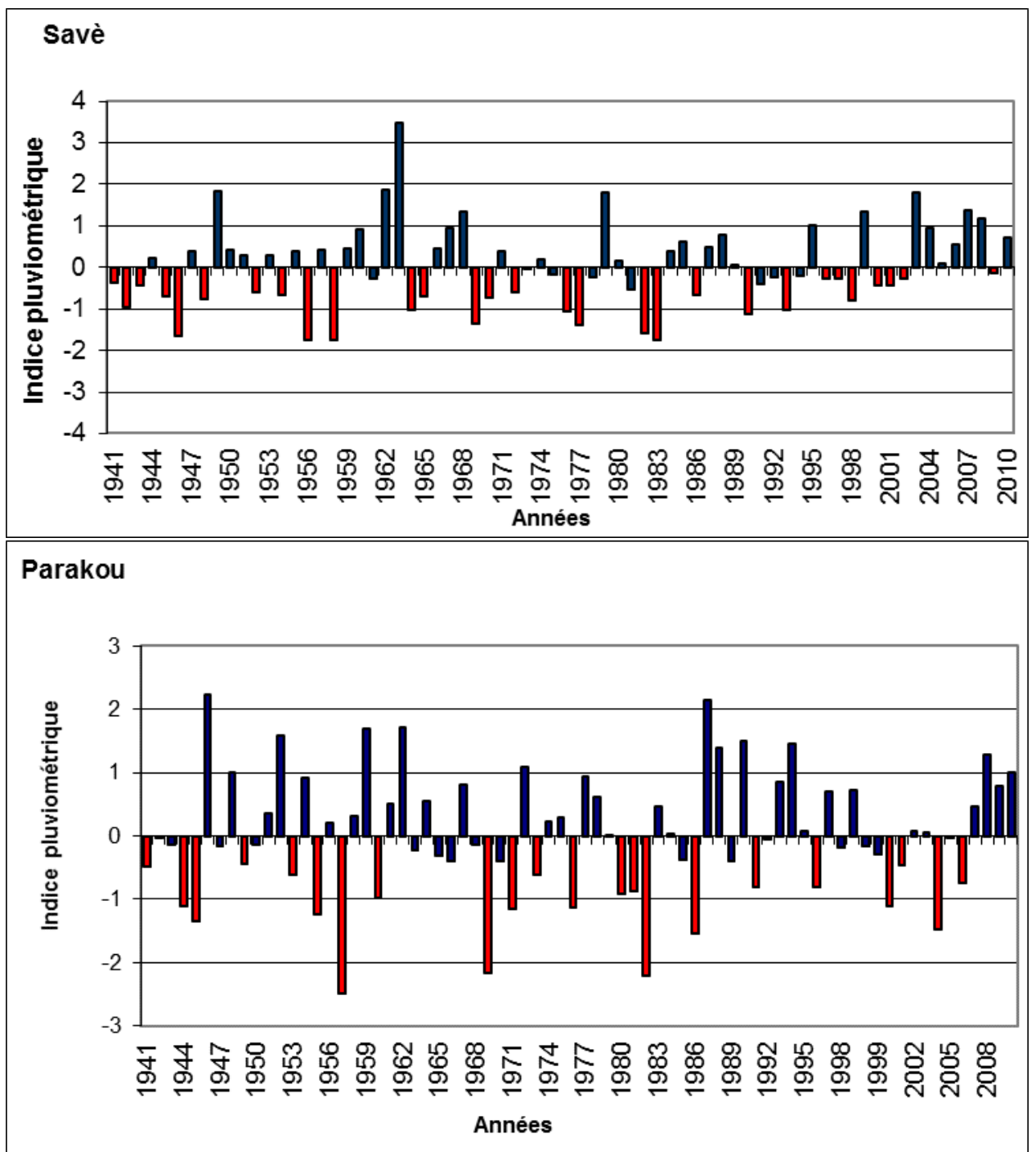

Figure 4: Variabilité pluviométrique interannuelle à Save et à Parakou. 

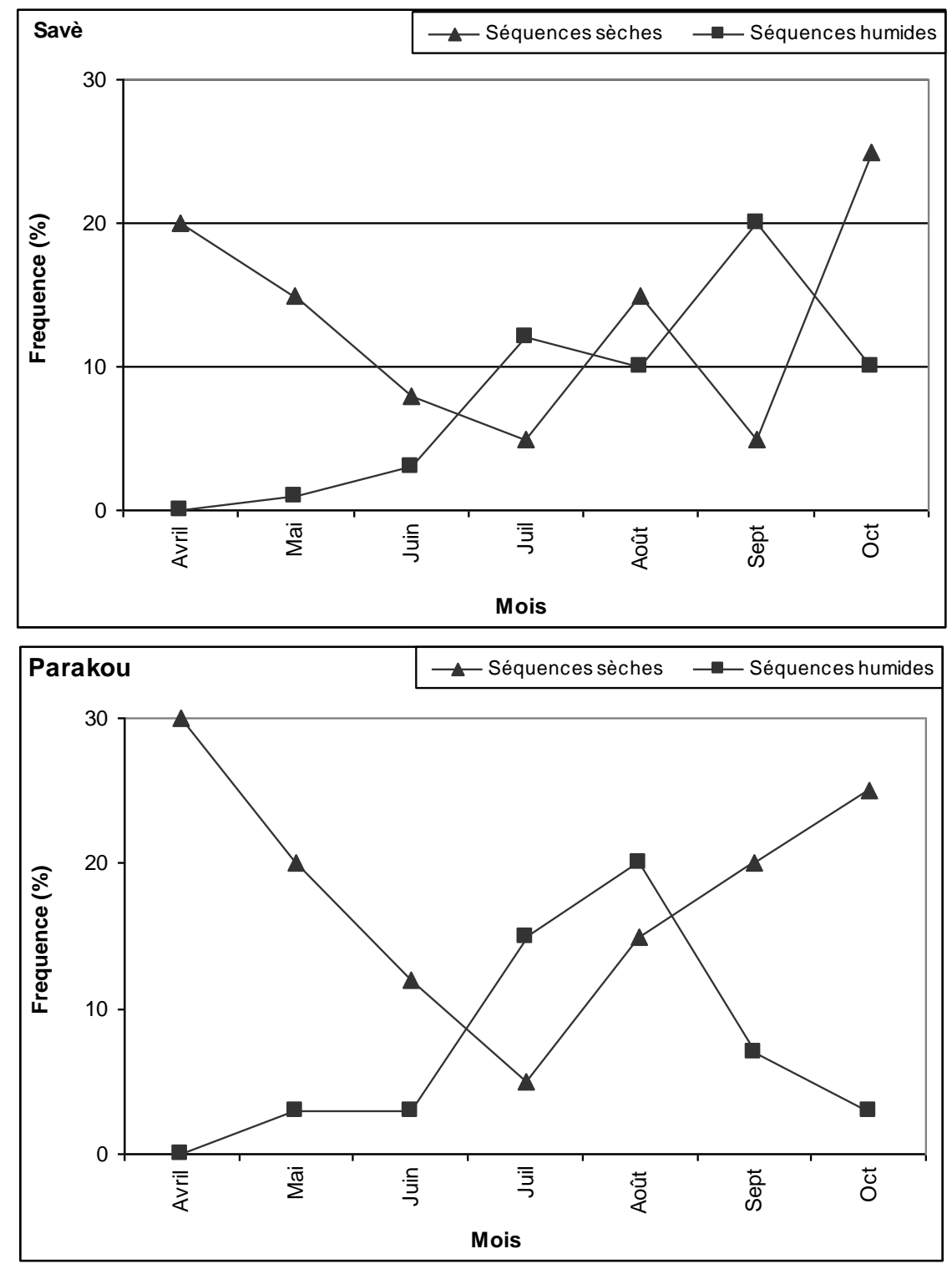

Figure 5: Fréquence des séquences sèches et humides au cours de la saison agricole.

Tableau 6: Calendrier des activités agricoles.

\begin{tabular}{|c|c|c|c|c|c|c|c|c|c|c|c|c|}
\hline & Jan & Fév & Mars & Avril & Mai & Juin & Juil & Août & Sept & Oct & Nov & Déc \\
\hline Saison sèch & & & & & & & & & & & & \\
\hline $\begin{array}{l}\text { Première } \\
\text { campagne } \\
\text { agricole }\end{array}$ & & & & & & & & & & & & \\
\hline $\begin{array}{l}\text { Deuxième } \\
\text { campagne } \\
\text { agricole }\end{array}$ & & & & & & & & & & & & \\
\hline
\end{tabular}




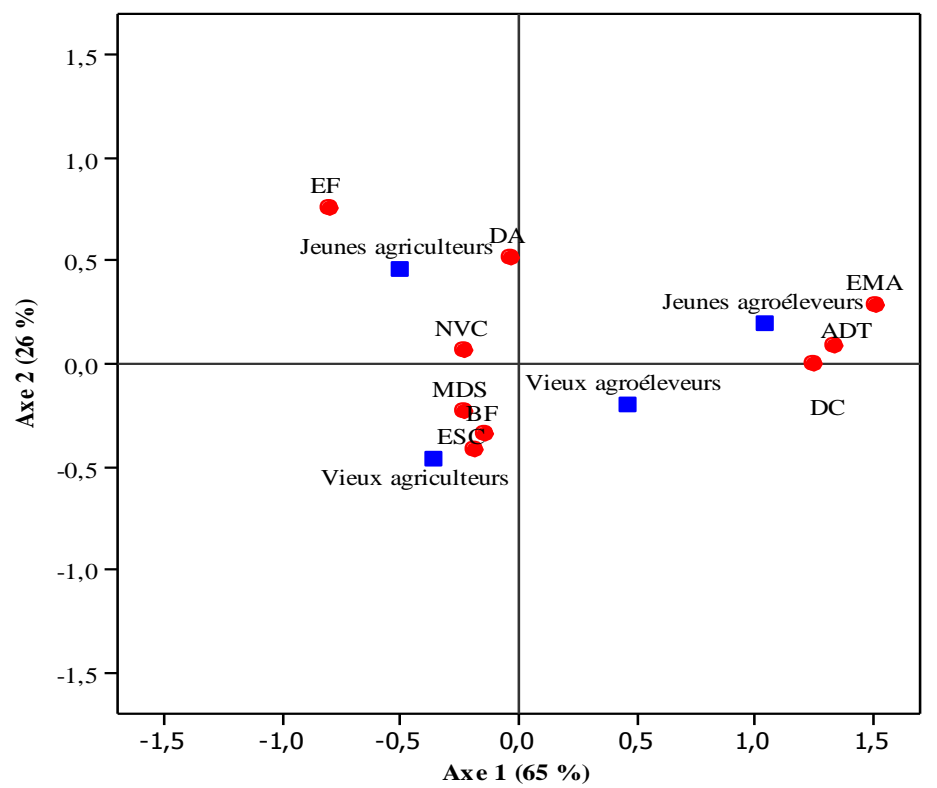

ESC: Extension de la surface cultivée, MDS : Modification des dates de semis, NVC : Choix de nouvelles variétés culturales, BF : Exploitation des bas-fonds, EF : Exploitation forestière, EMA : Emondage accru des arbres, ADT : Allongement de la durée de transhumance, DC : Déplacement des lieux de campement, DA : Diversification des activités.

Figure 6: Types d'adaptations par catégorie socioprofessionnelle.
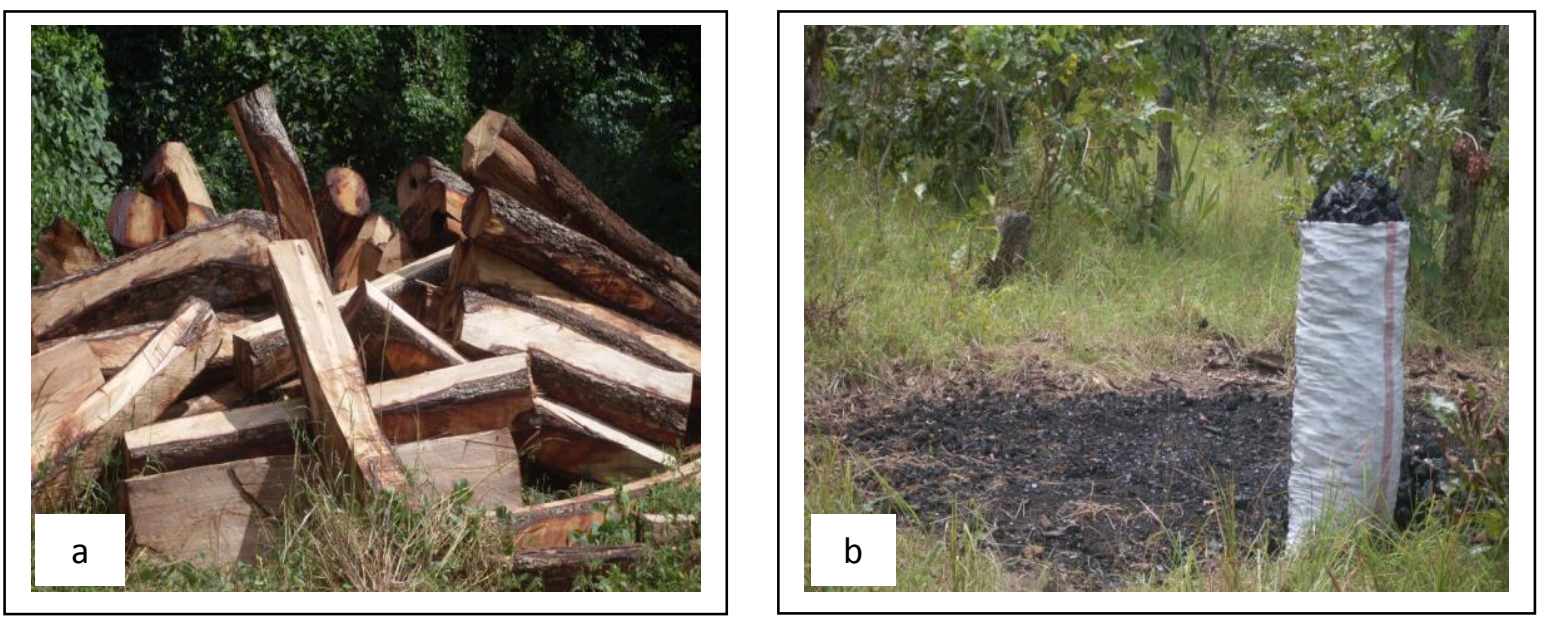

Figure 7: Madriers de bois entreposés en pleine brousse dans la Commune de Bassila (a) et Sacs de charbon de bois retrouvés en brousse dans la commune de Savè (b).

Prise de vue : Oloukoi, 2009.

\section{DISCUSSION}

Les paysans du centre du Bénin perçoivent les mutations pluviométriques à travers plusieurs aspects (démarrage et fin de la saison pluvieuse, occurrence des séquences sèches ou humides). Ce constat a été fait par Ogouwalé (2001), Yabi et Afouda (2007),
Yabi et Boko (2008), Issa (2012), qui ont travaillé sur la variabilité/changement climatique dans diverses régions du Bénin et sur différentes échelles (mensuelle, saisonnière et annuelle). De même, les perceptions évoquées, corroborent les travaux de Gnangle et al. (2009) sur la gestion des 
parcs à Karité dans la commune de Savè au Centre du Bénin. En effet, ces derniers ont non seulement montré que les paysans ont réellement leurs perceptions par rapport aux dernières mutations observées dans l'évolution du climat, mais ces perceptions épousent les tendances au niveau des données pluviométriques des stations météorologiques, ces dernières décennies (Loko et al., 2013). Mais les perceptions sont fonction du genre, de l'âge, de l'expérience et des groupes socioculturels de la gestion des parcs à karité au Centre Bénin. Aussi la présente étude complète les conclusions des travaux de Agbossou et al. (2012) qui ont pu relever que les populations des zones agro-écologiques les plus vulnérables du centre et du sud Bénin ont une lecture des phénomènes climatiques essentiellement fondée sur des savoirs localement construits.

Ouédraogo et al. (2010) ont fait un constat similaire dans toutes les trois zones agro-écologiques du Burkina-Faso. Cependant, en fonction des échelles spatiales d'analyses, des nuances apparaissent entre les perceptions communautaires et la réalité scientifique sur l'ampleur et la fréquence des mutations climatiques (Baudoin, 2010). Le rythme et l'intensité des anomalies perçues par les paysans sont souvent influencés par l'ampleur des conséquences (pertes de récoltes, pertes d'animaux) que par les statistiques rationnelles. De même, comme l'ont souligné Ogouwalé (2006), Yabi et Afouda (2007), les évènements historiques et socioéconomiques constituent les repères utilisés par les communautés paysannes du centre du Bénin pour situer la période des mutations climatiques. Dans ces conditions, des nuances, voire des différences, peuvent exister entre les dates indiquées par les paysans avec des dates indiquées par des mesures scientifiques.

Après une période de relative passivité et face à la persistance des mutations pluviométriques les paysans béninois ont commencé par développer des mesures adaptatives. C'est le cas par exemple des producteurs d'ananas au Sud du Bénin qui face aux impacts des mutations pluviométriques ont mis en œuvre plusieurs mesures adaptatives sur la base des savoirs empiriques et des conseils des techniciens agricoles. Ces mesures portent sur les semis multiples ou répétés, l'utilisation de la main-d'œuvre agricole salariée, l'utilisation des engrais chimiques, l'augmentation des superficies emblavées et les traitements phytosanitaires (Yabi, 2013). Toutefois il importe d'observer que les stratégies d'adaptation varient en fonction des exigences de chaque zone agro-écologique et selon les catégories de producteurs. La capacité d'un paysan à s'adapter aux changements des précipitations dépend de sa perception du phénomène, de la nécessité d'apporter des solutions et des possibilités qui lui sont offertes (Laube et al., 2011; Ouédraogo et al., 2010; Smit et al., 2006; Admassie et al., 2007 ). Dans tous les cas, les mesures visent à maintenir le niveau de production (végétale et animale) malgré l'hostilité des conditions climatiques ou diversifier les sources de revenus à travers la reconversion professionnelle (Brou, 2010). L'adoption spontanée de ces mesures témoigne de l'ingéniosité des paysans et de leur capacité à s'adapter aux contraintes naturelles. Cependant la généralisation de certaines mesures (exploitation forestière, exploitation des bas-fonds, émondage massif des arbres, extension des superficies agricoles) est susceptible d'engendrer des pressions sur les ressources naturelles et de fragiliser davantage les conditions de vie des populations. Il a été observé que certaines de ces mesures créent ou renforcent des déséquilibres au niveau de l'environnement. Par exemple le fait d'augmenter les emblavures agricoles renforce le système de l'agriculture itinérante sur brûlis qui présente des conséquences négatives sur l'environnement par la destruction des formations végétales denses dont les espèces 
sont détruites par feux de végétation, dessouchage ou écobuage. Cette pratique aboutit à une utilisation des terres, soit de manière incontrôlée ou de manière abusive avec des pratiques agricoles ou d'exploitation forestière devenues préoccupantes (Mama, 2002 ; Oloukoi, 2013 ; 2017).

\section{Conclusion}

Le présent travail a analysé les perceptions paysannes sur la variabilité pluviométrique au centre du Bénin et les stratégies d'adaptation. Il a permis de mieux comprendre que les variabilités pluviométriques ne sont pas seulement constatées par les scientifiques, mais perçues aussi par les paysans. Les perceptions mises en évidence ici sont basées sur le démarrage tardif, précoce ou sur la pluviosité excessive ou déficitaire d'une année à une autre. L'évolution de la pluviométrie en dent de scie n'est pas sans conséquence sur la production et la conduite des activités agro-pastorales. Bien que les perceptions paysannes soient subjectives, elles sont indicatives des années de déficits ou d'excédents pluviométriques telles que confirmées par les évidences scientifiques. Il est noté que des stratégies d'adaptation sont adoptées par des paysans et compte tenu de l'ampleur des perturbations de leurs activités par la variabilité pluviométrique, une majorité a opté pour l'augmentation des emblavures agricoles et la reconversion professionnelle. Cette reconversion professionnelle a contribué à l'émergence de nouvelles activités génératrices de revenus dont l'exploitation forestière par la production et la commercialisation du bois et du charbon de bois.

Des mesures d'accompagnement (formation, encadrement, financement) méritent d'être prises pour identifier et vulgariser les stratégies d'adaptation qui paraissent durables. Ces mesures sont nécessaires d'autant plus que les prévisions indiquent que la variabilité pluviométrique pourrait s'aggraver au cours des prochaines décennies en Afrique de l'ouest dans le cadre des changements climatiques dont les signes précurseurs sont déjà perceptibles. Dans cet ordre d'idée, il importe que les encadreurs du monde rural de même que les chercheurs, envisagent une révision officielle $\mathrm{du}$ calendrier agricole pour s'adapter aux modifications actuelles du climat.

\section{CONFLIT D'INTERETS}

Les auteurs déclarent que cet article ne fait pas l'objet d'un conflit d'intérêt

\section{CONTRIBUTIONS DES AUTEURS}

JO a conçu l'étude, collecté et analysé les données, et a rédigé le manuscrit. IY et CSH ont contribué à l'analyse des données, l'amélioration de l'approche méthodologique et l'orientation scientifique et la relecture de l'article.

\section{REMERCIEMENTS}

Les auteurs remercient les évaluateurs anonymes qui ont sensiblement contribué à l'amélioration de la qualité scientifique de cet article en consacrant leurs précieux temps à son évaluation critique.

\section{REFERENCES}

Adimi OSC, Oloukoi J, Tohozin CAB. 2018. Analyse spatiale multicritère et identification des sols propices à la production du maïs à Ouessè au Bénin. VertigO J.- la revue électronique en sciences de l'environnement [En ligne]. DOI:10.4000/vertigo.19885

Admassie A, Adenew B, Abebe T. 2007. Perceptions of stakeholders on climate change and adaptation strategies in Ethiopia. EEA Research Report, Addis Ababa.

Afouda F. 1990. L'eau et les cultures dans le Bénin central et septentrional : étude de la variabilité des bilans de l'eau dans leurs relations avec le milieu rural de la savane africaine. Thèse de Doctorat 
nouveau régime, Paris IV Sorbonne, 520 p.

Agossou DSM, Tossou CR, Vissoh VP, Agbossou KE. 2012. Perception des perturbations climatiques, savoirs locaux et stratégies d'adaptation des producteurs agricoles béninois. African Crop Science Journal, 20: 565-588.

Aho N, Ahloussou E, Agbahungba G. 2006. Evaluation concertée de la vulnérabilité aux variations actuelles du climat et aux phénomènes météorologiques extrêmes. Rapport de synthèse PANA-Benin / MEPNPNUD, Cotonou, 52p.

Atidegla SC, Bonou W, Agbossou EK. 2017. Relations entre perceptions des producteurs et surfertilisation en maraichage urbain et péri urbain au Bénin. Int. J. Biol. Chem. Sci., 11(5): 2106-2118.

DOI: http://dx.doi.org/10.4314/ijbcs.v11i5.14

Bodian A, Dacosta H, Dezetter A. 2011. Caractérisation spatio-temporelle du régime pluviométrique du haut bassin du fleuve Sénégal dans un contexte de variabilité climatique. Physio-Géo, 5: 107-124. DOI: 10.4000/physio-geo.1958

Baudoin MA. 2010. L'adaptation aux changements climatiques au sud du Bénin: Une analyse de la politique internationale et des besoins locaux. Geo-Eco-Trop., 34: 155 - 69.

Boko M, Niang I, Nyong A, Vogel C, Githeko A, Medany M, Yanda P. 2007. Africa Climate Change 2007: Impacts, Adaptation and Vulnerability. In Contribution of Working Group II to the Fourth Assessment Report of the Intergovernmental Panel on Climate Change, Parry ML, Canziani OF, Palutikof JP, van der Linden PJ, Hanson CE (Eds.). Cambridge University Press: Cambridge, UK; 433-467.

Boko M. 1988. Climat et communautés rurales du Bénin: Rythmes climatiques et rythme de développement. Thèse d'Etat ès lettre, Dijon, 607p.
Brou T. 2010. Variabilité' climatique, déforestation et dynamique agrodémographique en Côte d'Ivoire. Sécheresse, 21(4): 327-329. DOI: 10.1684/sec.2010.0266

Brou YT, Akindès F, Bigot S. 2005. La variabilité climatique en Côte d'Ivoire : entre perceptions sociales et réponses agricoles. Cahiers Agricultures, 14(6): 533-540.

Chabi OS, Tohozin CAB, Oloukoi J. 2018. Modélisation spatiale et évaluation multicritère dans la détermination des sites propices à la production du maïs à Ouèssè, Bénin. Int. J. Biol. Chem. Sci., 12(1): 253-265. DOI: https://dx.doi.org/10.4314/ijbcs.v12i1.20

Djenontin JA. 2010. Dynamique des stratégies et des pratiques d'utilisation des parcours naturels pour l'alimentation des troupeaux bovins au Nord-Est du Bénin. Thèse de Doctorat Unique, FSA, UAC, Benin, $275 \mathrm{p}$.

FAO, 2012. La transhumance transfrontalière en Afrique de l'Ouest Proposition de plan d'action. FAO, 146p.

FAO. 2000. Global Forest Resources Assessment 2000, Main Report. FAO Forestery paper, 140: 115-120.

Gnanglè CP, Yabi AJ, Glèlè Kakaï JLR, Sokpon N. 2009. Changements climatiques: Perceptions et stratégies d'adaptations des paysans face à la gestion des parcs à karité au CentreBénin. Bénin, 18 p.

IPCC. 2007. Climate Change 2007: Impacts, Adaptation and Vulnerability. Contribution of Working Group II to the Fourth Assessment Report of the Intergovernmental Panel on Climate Change. Cambridge University Press: Cambridge, UK.

INSAE. 2003. Troisième Recensement Général de la Population et de l'Habitation. INSAE, Cotonou, Bénin.

Kouassi AM, Kouamé KF, Koffi YB, Dje KB, Paturel JE, Oulare S. 2010. Analyse de la 
variabilité climatique et de ses influences sur les régimes pluviométriques saisonniers en Afrique de l'Ouest : cas du bassin versant du N'zi (Bandama) en Côte d'Ivoire. Cybergeo: European Journal of Geography, Environnement, Nature et Paysage, 513. DOI : 10.4000/cybergeo. 23388

Issa MS. 2012. Changements Climatiques et agrosystèmes dans le Moyen Bénin : impacts et stratégies d'adaptation. Thèse Unique de doctorat, EDP/FLASH/UAC, $273 p$.

Laube W, Schraven B, Awo M. 2011. Smallholder adaptation to climate change: Dynamics and limits in Northern Ghana. Climatic Change, $22 \mathrm{p}$.

Leeuwis C, Van den Band A. 2003. Communication for innovation. In agricultural and rural resource management. Building on the tradition of agricultural extension. Blackwell Science : Oxford ; 127-149.

Loko YL, Dansi A, Agre AP, Akpa N, Dossou-Aminon I, Assogba P, Dansi M, Akpagana K, Sanni A. 2013. Perceptions paysannes et impacts des changements climatiques sur la production et la diversité variétale de l'igname dans la zone aride du nordouest du Bénin. Int. J. Biol. Chem. Sci., 7(2): 672-695. DOI: http://dx.doi.org/10.4314/ijbcs.v7i2.23

Mama VJ. 2002. An integrated approach for land-use/cover change analysis in a central region of Benin Republic. PhD Thesis in Geography, University of Lagos, Lagos, 221 p.

Odjugo PAO. 2011. Climate change and global warming: the Nigerian perspective. Journal of Sustainable Development and Environmental Protection, 1(1): 6-17.

Ogouwalé E. 2001.Vulnérabilité/Adaptation de l'agriculture aux changements climatiques dans le Département des
Collines. Mémoire de maîtrise de Géographie, DGAT/FLASH/UAC, 119p.

Ogouwalé E. 2006. Changements climatiques dans le Bénin méridional et central : Indicateurs, Scénarios et Prospective de la Sécurité Alimentaire. Thèse de doctorat Unique Option dynamique des Systèmes Climatiques, EDP/ FLASH/ UAC, Abomey-Calavi, $302 \mathrm{p}$.

Oloukoi J, Mama VJ. 2017. Analyse des facteurs et impacts de l'exploitation des bas-fonds au centre du Bénin. Revue de Géographie du Bénin (BenGeo), 21: 4872.

Oloukoi J. 2017. Rate of land cover change and its determinants in the soudanoguinea transition zone of Benin Republic. Ethiopian Journal of Environmental Studies \& Management, 10(5): 691-704. DOI: https://dx.doi.org/10.4314/ejesm.v10i5.1 2

Oloukoi J. 2013. Scénario socio-économique et écologique des changements de l'occupation des terres au Bénin », VertigO - la revue électronique en sciences de l'environnement, 13(1): 1-22. DOI : 10.4000/vertigo.13267

Oloukoï J. 2012. Utilité de la télédétection et des systèmes d'information géographique dans l'étude de la dynamique spatiale de l'occupation des terres au centre du Bénin. Thèse Unique de doctorat, EDP/FLASH/UAC, Abomey-Calavi, 304p.

OMM. 2012. Guide d'utilisation de l'indice de précipitations normalisé. OMM-N ${ }^{\circ}$ 1090, Genève (Suisse), 25p.

Orekan V. 2007. Implementation of the local land-use and land-cover change model CLUE-s for Central Benin by using socio-economic and remote sensing data. PhD Thesis, University of Bonn, 204 p.

Ouoba HY, Bastide B, Coulibaly-Lingani P, Kabore SA, Boussim JI. 2018. Connaissances et perceptions des producteurs sur la gestion des parcs à 
Vitellaria paradoxa C. F. Gaertn. (Karité) au Burkina Faso. Int. J. Biol. Chem. Sci., 12(6): 2766-2783. DOI: https://dx.doi.org/10.4314/ijbcs.v12i6.23

Ouoba AP. 2013. Changements climatiques, dynamique de la végétation et perception paysanne dans le Sahel burkinabè. Thèse de Doctorat Unique de Géographie, Université de Ouagadougou, BurkinaFaso, 305p.

Ouédraogo M, Dembélé Y, Somé L. 2010. Perceptions et stratégies d'adaptation aux changements des précipitations : cas des paysans du Burkina Faso. Sécheresse, 21(2): 87-96. DOI: 10.1684/sec.2010.0244

Puget JL, Blanchet R, Salencon J, Carpentier A. 2010. Le changement climatique. Rapport Académie des Sciences, 21 p.

Ruault C. 2007. L'enquête sociotechnique dans une perspective compréhensive : 1 . Fondements et principes méthodologiques. Note de cours du Module de Master supagro IRC GERDAL-IRAM.

Sarr MA. 2008. Variabilité pluviométrique en Afrique de l'Ouest : dynamique des espaces végétaux à partir des images satellitales. Exemple du bassin versant du Ferlo (Sénégal). Actes Journées de Climatologie 'Climat et société : Climat et végétation', Nantes (France), 57-76.

Smit B, Wandel J. 2006. Adaptation, adaptive capacity and vulnerability. Global Environmental Change, 16: 282-292. DOI:

https://doi.org/10.1016/j.gloenvcha.2006 .03 .008

Yabi I. 2013. Perceptions paysannes des facteurs de risques climatiques sur la production de l'ananas à Toffo au Bénin. Revue de Géographie Tropicale et d'Environnement, 1:84-95.

Yabi I. 2008. Etude de l'agroforesterie à base d'anacardier et des contraintes climatiques à son développement dans le
Centre du Bénin. Thèse de doctorat nouveau régime, Universite d'Abomey Calavi, Bénin, 240 p.

Yabi I, Afouda F. 2007. Variabilité pluviométrique du début de la saison agricole et mesures d'adaptation dans le département des Collines au Bénin (Afrique de l'ouest). Actes du 1er colloque de l'UAC des Sciences, Cultures et Technologies, Géographie, Abomey-Calavi, Bénin, 315-327.

Yabi I, Boko M. 2008. Recherche sur le démarrage de la saison pluvieuse dans le Département du Borgou au Bénin (Afrique de l'Ouest). Actes du XXIème colloque de l'Association Internationale de Climatologie, Montpellier, France, 673-678.

Yabi I, Afouda F, Ogouwalé E, Boko M. 2011. Années pluviométriques extrêmes et incidences socio environnementales dans une région de montagne : le Département de l'Atacora au Bénin. Actes du XXIVème Colloque de l'Association Internationale de Climatologie, Roverto (Italie), 597-602.

Yegbemey RN, Yabi JA, Aïhounton, GB, Paraïso A. 2014. Modélisation simultanée de la perception et de l'adaptation au changement climatique: cas des producteurs de maïs du Nord Bénin (Afrique de l'Ouest). Cahiers Agricultures, 23: 177-87, DOI: https://doi.org/10.1684/agr.2014.0697

Zakari S, Yabi I, Ogouwalé E, Boko M. 2012. Analyse de quelques caractéristiques de la saison des pluies dans le Département du Borgou (Bénin, Afrique de l'Ouest). Actes du XXVème Colloque de l'AIC, Grenoble, France, 693-698. 